\title{
A Decrementing Form of Plasticity Apparent in Cerebellar Learning
}

\author{
Tatsuya Ohyama, ${ }^{1}$ Horatiu Voicu, ${ }^{3}$ Brian Kalmbach, ${ }^{1}$ and Michael D. Mauk ${ }^{1,2}$ \\ ${ }^{1}$ Center for Learning and Memory and ${ }^{2}$ Section of Neurobiology, The University of Texas at Austin, Austin, Texas 78712-0805, and ${ }^{3}$ Department of \\ Neurobiology and Anatomy, The University of Texas-Houston Health Science Center, Houston, Texas 77030
}

Long-term synaptic plasticity is believed to underlie the capacity for learning and memory. In the cerebellum, for example, long-term plasticity contributes to eyelid conditioning and to learning in eye movement systems. We report evidence for a decrementing form of cerebellar plasticity as revealed by the behavioral properties of eyelid conditioning in the rabbit. We find that conditioned eyelid responses exhibit within-session changes that recover by the next day. These changes, which increase with the interstimulus interval, involve decreases in conditioned response magnitude and likelihood as well as increases in latency to onset. Within-subject comparisons show that these changes differ in magnitude depending on the type of training, arguing against motor fatigue or changes in motor pathways downstream of the cerebellum. These phenomena are also observed when stimulation of mossy fibers substitutes for the conditioned stimulus, suggesting that changes take place within the cerebellum or in downstream efferent pathways. Together, these observations suggest a plasticity mechanism in the cerebellum that is induced during training sessions and fades within $23 \mathrm{~h}$. To formalize this hypothesis more specifically, we show that incorporating a short-lasting potentiation at the granule cell to Purkinje cell synapses in a computer simulation of the cerebellum reproduces these behavioral effects. We propose the working hypothesis that the presynaptic form of long-term potentiation observed at these synapses is reversed by time rather than by a corresponding long-term depression. These results demonstrate the utility of eyelid conditioning as a means to identify and characterize the rules that govern input to output transformations in the cerebellum.

\section{Introduction}

Forms of plasticity such as long-term potentiation (LTP) and long-term depression (LTD) are of interest for their potential to explain long-term storage of memories (Maren and Baudry, 1995; Cruikshank and Weinberger, 1996; Martin et al., 2000; Ito, 2001; Martin and Morris, 2002; Lynch, 2004). In contrast, shortterm plasticity lasting hundreds of milliseconds to tens of seconds has been connected to short-term learning (Zucker, 1989; Stopfer and Carew, 1996; Fisher et al., 1997; Zucker and Regehr, 2002), and to temporal processing (Abbott and Nelson, 2000; Buonomano, 2000; Fortune and Rose, 2000, 2002; Mauk and Buonomano, 2004; Karmarkar and Buonomano, 2007; Buonomano and Maass, 2009). We present evidence for a decrementing form of plasticity in the cerebellum that falls between long-term and short-term plasticity. We characterize within-session changes in the properties of conditioned eyelid responses that reverse by the beginning of the next day. Such changes are apparent but have not been systematically analyzed in previously published eyelid conditioning studies in rat [Stanton et al., 1992 (their Figs. 2, 3, 5); Kehoe and White, 2002 (their Fig. 1)]; Kehoe et al., 2004 (their Figs. 1, 2)] and rabbit [Weidemann and Kehoe, 2005 (their Fig. 3)] (see also Kehoe and Gormezano, 1974).

\footnotetext{
Received May 13, 2010; revised 0ct. 12, 2010; accepted 0ct. 17, 2010.

This work was supported by National Institute of Mental Health Grants MH74006, MH46904, and MH57051 to M.D.M.

Correspondence should be addressed to Michael D. Mauk, Center for Learning and Memory, The University of Texas at Austin, 1 University, Station Stop C7000, Austin, TX 78712-0805. E-mail: mike@dm.utexas.edu. DOI:10.1523/JNEUROSCI.2455-10.2010

Copyright $\odot 2010$ the authors $\quad 0270-6474 / 10 / 3016993-11 \$ 15.00 / 0$
}

The ability to infer cerebellar processes from the behavioral properties of eyelid conditioning stems from the relatively direct way that eyelid conditioning engages the cerebellum (Mauk and Donegan, 1997; Medina and Mauk, 2000; Ohyama et al., 2003). Eyelid conditioning involves paired presentations of a conditioned stimulus [(CS) usually a tone] with a reinforcing unconditioned stimulus (US) such as periorbital stimulation. With training, the CS acquires the ability to elicit a conditioned eyelid response. The CS and US are conveyed to the cerebellum via activation of mossy and climbing fiber inputs, respectively, and cerebellar output drives the expression of conditioned responses (CRs) (McCormick and Thompson, 1984; Thompson and Steinmetz, 2009). The evidence for this arises in part from the ability to substitute stimulation of mossy fiber inputs for the tone CS to support robust learning (Steinmetz et al., 1989; Hesslow et al., 1999). Along with similar data for the US and the climbing fiber input (Mauk et al., 1986), these observations illustrate how eyelid conditioning reveals learning in the cerebellum that alters the transformation of mossy fiber inputs into cerebellar output.

We demonstrate that conditioned eyelid responses show systematic within-session changes that reverse by the next day. These changes - a decrease in response likelihood/magnitude and an increase in latency to onset-increase monotonically with the interstimulus interval (ISI). Within-subject comparisons show that the magnitude of these changes differs depending on the type of training, ruling out changes in the motor pathways downstream of the cerebellum. Since the ability of mossy fiber stimulation to support learning with similar properties excludes 
upstream processes, these data suggest the operation of a decrementing plasticity process within the cerebellum that lasts for more than tens of minutes but $<23 \mathrm{~h}$.

Simulation results illustrate how these observations may be explained by a decrementing potentiation $(\mathrm{dP})$ at the granule cell to Purkinje cell synapses in the cerebellar cortex. From these findings, we propose the working hypothesis that the presynaptically expressed form of LTP at these synapses (Salin et al., 1996) may be reversed by time. Regardless, our data suggest that a decrementing plasticity process exists in the cerebellum and that its contributions must be factored into a full understanding of the cerebellum.

\section{Materials and Methods}

Subjects and surgery. Two hundred and one naive male New Zealand albino rabbits, each weighing $2.5-3.0 \mathrm{~kg}$, served as the subjects. They were housed in individual cages, maintained on a fixed daily diet, and given water ad libitum. Treatment of animals and surgical procedures were in accordance with an institutionally approved animal welfare protocol at the University of Texas Health Science Center in Houston and later at the University of Texas at Austin.

Subjects were preanesthetized with $5 \mathrm{mg} / \mathrm{kg}$ acepromazine and 40 $\mathrm{mg} / \mathrm{kg}$ ketamine, and their skulls were immobilized in a stereotaxic restrainer. Anesthesia was maintained with isofluorene (1-2\% mixed in oxygen) throughout the surgery. A $2 \mathrm{~cm}$ incision exposed the skull, and four holes were drilled to accommodate small screws for fixing the head stage. In some animals, stimulating electrodes were implanted in the middle cerebellar peduncle $(3.0 \mathrm{~mm}$ anterior-posterior, $5.5 \mathrm{~mm}$ mediallateral, and $16 \mathrm{~mm}$ dorsal-ventral from lambda). A large head bolt for attaching the infrared recording device was then secured with dental acrylic together with the implant, and the wound was loosely sutured. Finally, two stainless steel stimulating electrodes were implanted subdermally $0.5 \mathrm{~cm}$ rostral and caudal to the left eye. Analgesics and antibiotics were administered for $2 \mathrm{~d}$ following surgery. Subjects were allowed at least 1 week to recover before training began.

Apparatus. Two custom-designed chambers were used to train animals. Each was equipped with a speaker connected to an audio source module that generated tones (model V-85, Coulbourn Instruments) and a pair of isolated pulse stimulators (model 2100, A-M Systems) that delivered electrical pulses through the periorbital electrodes. For experiments using mossy fiber stimulation as a substitute for the CS, $500 \mathrm{~ms}$ trains of pulses $(50 \mathrm{~Hz}, 0.1 \mathrm{~ms}$ constant current cathodal pulses, $100 \mu \mathrm{A})$. An infrared emitter/detector system attached to the head stage recorded movement of the left external eyelid by detecting changes in the amount of reflected infrared light. The output of these detectors has been confirmed to be linearly related to eyelid position $\pm 5 \%$. The detector was calibrated at the start of each daily training or test session by eliciting full eyelid closure (by delivering a US or manually touching near the eye). The voltage-to-millimeter relationship was then determined assuming full eyelid closure to be $6 \mathrm{~mm}$. Stimulus presentation and response measurement were implemented by custom software interfaced via National Instruments $\mathrm{A} / \mathrm{D}$ and counter/timer boards.

Conditioning procedure. For most experiments, the CS was a tone: 1 or $9.5 \mathrm{kHz} ; 85 \mathrm{~dB} ; 70 \mathrm{~dB}$ background noise. For stimulation of mossy fibers as the CS, pulse trains were applied through electrodes implanted in the middle cerebellar peduncle ( 50 or $100 \mathrm{~Hz}, 40 \mu$ s pulse width, 100-150 $\mu \mathrm{A}$, train length set to mimic tone length). Stimulation through electrodes implanted in the middle cerebellar peduncle reduces the chances of unwanted activation of fibers of passage as all axons in this fiber tract are mossy fibers projecting to the cerebellum. The US was a train of current pulses ( $100 \mathrm{~Hz}, 1 \mathrm{~ms}$ pulse width, $1.0-2.5 \mathrm{~mA}, 50 \mathrm{~ms}$ ) delivered through the periorbital electrodes. The intensity was adjusted for each animal to be above that required to elicit full eyelid closure but below threshold for eliciting robust head turns and other responses potentially indicative of pain. Training sessions consisted of 12 blocks, each containing 8 paired CS-US trials and 1 CS-alone probe trial. On paired trials, US onset followed CS onset by ISIs ranging from 150 to $1050 \mathrm{~ms}$, depending on the experiment, and in each case the CS lasted until $50 \mathrm{~ms}$ beyond US onset. On CS-alone trials, the CS lasted the same duration as the sister paired trials, and the US was omitted. The mean intertrial interval ranged pseudorandomly from 20 to $40 \mathrm{~s}$, with a mean of $30 \mathrm{~s}$. Responses were sampled at a rate of $1 \mathrm{kHz}$, and $2500 \mathrm{~ms}$ sweeps consisting of $200 \mathrm{~ms}$ before and $2300 \mathrm{~ms}$ after CS onset were collected for each trial and stored for subsequent offline analysis using custom software.

One set of animals was trained with delay and trace conditioning trials intermixed, a protocol that allows within-subject analyses of the circuitry and mechanisms underlying delay and trace eyelid conditioning (Kalmbach et al., 2009). On half of the trials, the CS was a $550 \mathrm{~ms}$ tone (1 or $9.5 \mathrm{kHz})$ that coterminated with the 50 ms US (delay trials). On the other half of the trials (trace trials), the CS was a $500 \mathrm{~ms}$ tone of the alternative frequency $(9.5$ or $1 \mathrm{kHz}$ ) whose offset was followed $500 \mathrm{~ms}$ later by the US (i.e., a $1000 \mathrm{~ms}$ ISI).

Data analysis. A conditioned response was defined as an eyelid response (closure) of at least $0.3 \mathrm{~mm}$ occurring $>40 \mathrm{~ms}$ after CS onset and before the US (on paired trials) or before the end of the trial (for CS-alone probes). The peak conditioned response amplitude was defined as the maximum value attained within these time periods compared with the average eyelid position in the $200 \mathrm{~ms}$ before CS onset. Several measures including latency to onset (time to first inflection of the response), latency to criterion (the time at which eyelid closure attained the criterion amplitude of $0.3 \mathrm{~mm}$ ), and CR magnitude (the peak amplitude of the responses excluding trials where the response did not meet the $0.3 \mathrm{~mm}$ criterion) were calculated using custom software. In addition, the unconditioned response (UR) amplitude was obtained from reinforced trials.

Statistical testing of differences in means was conducted by repeatedmeasures and mixed ANOVA. Post hoc tests were conducted using the Tukey test, $F$ test for simple effects, and paired $t$ tests where appropriate.

Computer simulation of the cerebellum. Simulation results came from a large-scale computer simulation of the cerebellum, based on the original simulations of Buonomano and Mauk (1994), Medina and Mauk (1999), and Medina et al. $(2000,2001,2002)$, that reproduced the main behavioral properties of eyelid conditioning including acquisition and extinction. The simulation is composed of 13,878 cells and 194,480 synapses. Each neuron is simulated using a single-compartment, leaky, integrateand-fire model in which synaptic, but not active, conductances are represented. Cellular properties related to active conductances (e.g., absolute and relative refractory periods) were captured by a varying threshold that could depend on recent spiking activity. Synaptic conductances are represented as step increases upon activation and exponential decay with time constants based on observations from cerebellar slice studies (Shinoda et al., 1987; Konnerth et al., 1990; Midtgaard, 1992; Gould et al., 1993; Barbour, 1993; Llano and Gerschenfeld, 1993; Puia et al., 1994; D’Angelo et al., 1995; Dieudonné, 1995, 1998; Kaneda et al., 1995; Mouginot and Gähwiler, 1995; Virginio et al., 1995; Brickley et al., 1996; Lang et al., 1996; Simpson et al., 1996; Vincent and Marty, 1996; Best and Regehr, 2009). When relevant (e.g., granule cells), neurons have separate representations of AMPA and NMDA glutamate-activated synaptic conductances as well as GABA-mediated inhibitory conductances. Synaptic and leak conductances are used to update the membrane potential with a time step of $1 \mathrm{~ms}$. The membrane potential calculations follow the general form:

$$
\begin{aligned}
\Delta V_{m}=\left[g_{\text {Leak }}\left(E_{\text {Leak }}-V_{m}\right)+g_{E}\left(E_{\text {Exite }}\right.\right. & \left.-V_{m}\right) \\
& \left.+g_{i}\left(E_{\text {Inhibit }}-V_{m}\right)\right] / C \mathrm{C},
\end{aligned}
$$

where $\Delta V_{m}$ is membrane potential, $g_{\text {Leak }}$ is leak conductance, $g_{E}$ and $g_{i}$, respectively, are excitatory and inhibitory conductances, $E_{X}$ is the relevant equilibrium potential, and $C_{m}$ is membrane capacitance.

Connectivity generally follows the geometric relationships as well as divergence and convergence ratios of synaptic connections between cells types. Table 1 shows the number of neurons and the divergence and convergence ratios of each cell type represented in the simulation. The granule cells are assumed to be arranged in a virtual two-dimensional $120 \times 100$ array, with other cells arranged on this grid according to their relative densities. The steps involved in establishing the connections of one granule cell onto the Golgi cell layer serves as an illustration for how the connectivity of the entire network was established. The geometry of 
Table 1. Connectivity in the computer simulation

\begin{tabular}{lll}
\hline Cell type (No.) & Inputs: source $(+/-):$ No. & No. of outputs \\
\hline Mossy fibers (600) & NA & To granule cells $(+): 80$ \\
Climbing fibers (4) & From deep nucleus $(-): 2$ & To deep nucleus cells $(-): 2$ \\
Granule (12,000) & From mossy fibers $(+): 4$ & To Purkinje $(+): 1$ \\
& From Golgi cells $(-): 4$ & To basket $(+): 1$ \\
& & To stellate $(+): 1$ \\
Golgi (900) & From mossy fibers $(+): 4$ & To granule $(-): 53$ \\
& From granule cells $(+): 64$ & \\
UBC (6) & NA & To granule $(+): 1$ \\
Basket (96) & From granule cells $(+): 125$ & To Purkinje $(-): 4$ \\
Stellate (240) & From granule cells $(+): 50$ & To Purkinje $(-): 1$ \\
Purkinje (24) & From granule cells $(+): 500$ & To deep nucleus cells $(-): 2$ \\
& From stellate cells $(-): 10$ & \\
Deep nucleus (8) & From basket cells $(-): 16$ & \\
& From mossy fibers $(+): 75$ & To climbing fibers $(-): 1$ \\
\hline
\end{tabular}

NA, Not applicable; UBC, unipolar brush cell.

the granule cell axons (T-shaped, straight, and relatively long) are used to specify the geometric extent over which the granule cell could conceivably make a synapse with a Golgi cell. For granule cell synapses onto Golgi cells, this is a long, thin rectangle whose long side is determined by the length of granule cell axons (the parallel fibers) and whose short side is determined by the diameter of Golgi cell dendritic trees. Golgi cells within this rectangle are candidate targets for this granule cell. The number of candidate Golgi cells that is selected is based on the divergence ratio of granule to Golgi connections. Should a connection exceed the convergence ratio of granule cells for that Golgi cell, a different Golgi cell is randomly selected and the process is repeated. Thus, by specifying the geometric range of connections, divergence ratios and convergence ratios, the entire network can be wired following this algorithm.

Long-term synaptic plasticity is represented at two classes of synapses. The granule cell to Purkinje cell synapses undergo LTD and LTP as controlled by climbing fibers (Sakurai, 1987; Hirano, 1990; Linden, 1994; Ito, 2001; Lev-Ram et al., 2002, 2003; Safo and Regehr, 2005, 2008). The mossy fiber-to-deep nucleus synapses undergo LTP and LTD as controlled by Purkinje cells (Miles and Lisberger, 1981; Medina and Mauk, 1999; Pugh and Raman, 2006, 2008, 2009; Zhang and Linden, 2006). To implement these forms of plasticity, the following was performed every fifth time step. Active granules to Purkinje synapses were reduced in strength if their activity fell within a $100 \mathrm{~ms}$ time window preceding a climbing fiber input and were otherwise increased in strength. Each active mossy fiber-to-nucleus synapse was increased in strength if its activity occurred in a time window following an abrupt pause in Purkinje activity and decreased in strength when its activity occurred during strong Purkinje cell inhibitory input.

For simulations implementing $\mathrm{dP}$ at the granule to Purkinje synapses, one of the following two procedures was used. At each time step an eligibility variable $\left(E_{i}\right)$ was updated for each granule to Purkinje synapse:

$$
\begin{gathered}
\text { If spike }=1 \text {, then } E_{i}=E_{i} \times 2.5 \\
\text { If spike }=0 \text {, then } E_{i}=E_{i}-\left(\left(E_{i}-\text { Base }\right) \times 0.0048\right),
\end{gathered}
$$

where Base is the baseline value of $E_{\mathrm{i}}(0.002)$ and the value 0.0048 produced exponential decay back to Base with a time constant of $20 \mathrm{~ms}$. This mimicked a nonlinear, activity-dependent buildup of eligibility. When this variable exceeded the threshold of 1.0 , which generally required a burst of spikes, $E_{i}$ was reset to Base and the $\mathrm{dP}$ value of the synapses was incremented by 0.00025 , up to a maximum value that ranged from 0.075 to 0.15 (see Results). This $\mathrm{dP}$ value was added to the existing strength of the granule to Purkinje synapse (which ranged from 0.0 to 1.0 ) to determine the final strength of the synapse each time bin. The $\mathrm{dP}$ values decreased gradually to zero during the between-sessions background activity runs to mimic the decrementing nature of the plasticity. Two forms of this $\mathrm{dP}$ were implemented with similar results: one that was indepen- dent of climbing fiber inputs as described above, and one where induction of $\mathrm{dP}$ was prevented for time bins within a $100 \mathrm{~ms}$ interval following a climbing fiber input to the Purkinje cell.

Mossy fibers were assigned a range of average firing rates, which then determined the frequency at which noisy excitatory conductances were activated. This gives the simulation a noisy and varied background level of activity. Presentation of a CS is emulated by changing the firing rate of 15 of the 600 mossy fibers. Nine were made to fire phasically at the onset of the CS, and for the remaining six activity was increased tonically for the duration of the CS (Boyd and Aitkin, 1976; Aitkin and Boyd, 1978). Presentation of the US was emulated by applying a brief excitatory conductance to the climbing fiber. The spiking activity of the eight nucleus cells was integrated over a $20 \mathrm{~ms}$ time span to represent the output of the simulation.

Before training, the simulation is run with background mossy fiber inputs for at least five million time steps to allow synaptic weights and the activity of the network to achieve equilibrium. Training trials were delivered the equivalent of once every $5 \mathrm{~s}$. To emulate training procedures in the rabbit, trials were delivered in groups of 108, and then 5,000,000 time bins were run with background inputs to mimic activity between training sessions. In simulations where this was relevant, the $\mathrm{dP}$ at granule to Purkinje synapses gradually reversed to zero during these background sessions. The state of the network (e.g., synaptic weights, conductances) could be saved for subsequent analyses.

\section{Results}

\section{Reversible, within-session changes in conditioned eyelid responses}

The initial observations suggesting a decrementing plasticity process in the cerebellum came from an analysis of a relatively large dataset $(n=33)$ of animals given delay conditioning for $10 \mathrm{~d}$ using an interstimulus interval of $500 \mathrm{~ms}$ (D500 group). These data revealed that at asymptotic levels of performance there are within-session changes in responding that reverse between sessions (Fig. 1, D500). Specifically, the likelihood of conditioned responses (percentage of CRs) and the magnitude (average amplitude of responses that met the conditioned response criterion) of the conditioned responses both showed clear within-session decreases. In addition, measures of response timing such as latency to onset showed within-session increases that appeared to reverse by the next session. Together, these changes produced a characteristic porpoising of the response measures that is readily apparent in Figure 1.

A similar analysis of animals from two different groups revealed a pattern of changes even more robust than that seen for delay 500. For these two groups, a longer ISI (1000 ms) was used: D1000 group $(n=18)$ involved delay conditioning, and T1000 group $(n=33)$ involved trace conditioning using a 500 ms CS paired with the US $500 \mathrm{~ms}$ after CS offset (for a total ISI of $1000 \mathrm{~ms}$ ). Both groups displayed larger within-session changes than the D500 group. For all three groups, the amplitude of the reflex URs elicited in each trial by the US appeared to be stable.

To quantify and statistically evaluate these phenomena more precisely, we focused our analysis on the data from the last $5 \mathrm{~d}$ of training in each group. To ensure that these $5 \mathrm{~d}$ represented asymptotic performance, we conducted nine separate repeatedmeasures ANOVAs (for the three groups and three response measures) examining the average of the first block over these five sessions. None of these tests showed significant differences, indicating that each response measure for each group began each session at essentially the same value. To evaluate the withinsession changes, each data point for these analyses was the normalized value for each block of nine trials (value for a block divided in each case by the value for the first block for that animal 

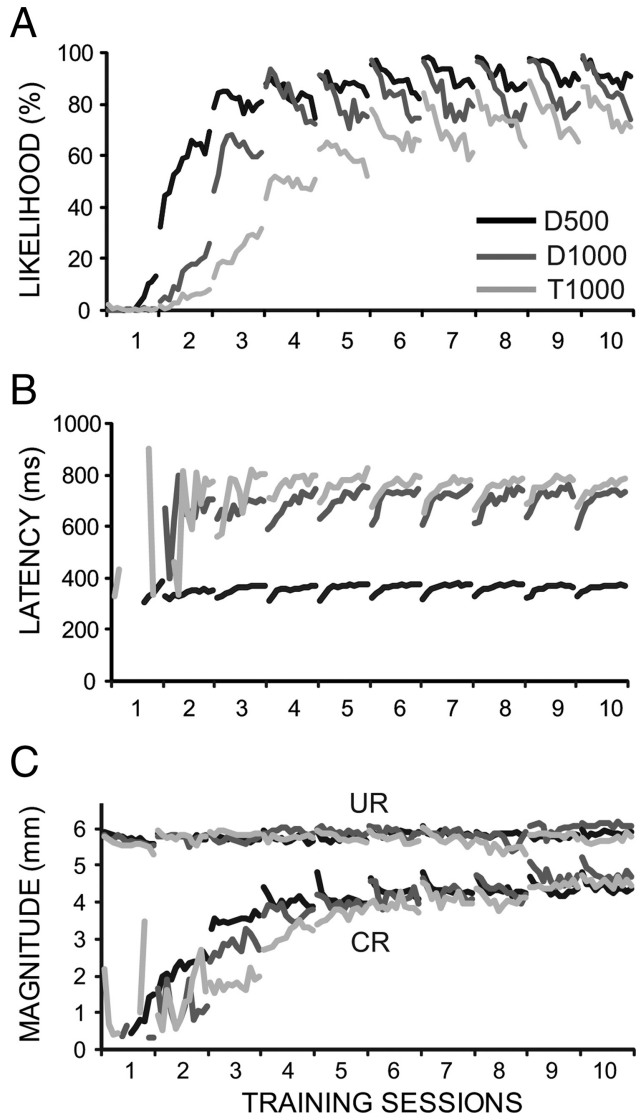

Figure 1. Evidence from three groups of animals for within-session changes in conditioned eyelid responses that reverse between sessions. Two groups of rabbits were trained with delay conditioning using an ISI of $500 \mathrm{~ms}(\mathrm{D} 500, n=33)$ and $1000 \mathrm{~ms}$ (D1000, $n=18)$, and a third group was trained with trace conditioning using a $500 \mathrm{~ms} C S$ and a $500 \mathrm{~ms}$ trace interval for a total ISI of $1000 \mathrm{~ms}(\mathrm{~T} 1000, n=33)$. A, B, Ten daily training sessions reveal that at asymptotic levels of performance (here, days $6-10$ ) there are within-session decreases in response likelihood $(\boldsymbol{A})$ and increases in latency to onset $(\boldsymbol{B})$. $\boldsymbol{C}$, more subtle within-session decreases are shown in response magnitude, which is the average amplitude of the responses that met the criterion for a conditioned response (i.e., excluding nonconditioned responses). For all three response measures, the within-session changes reverse between sessions.

and session). These normalized scores were then averaged across the last $5 \mathrm{~d}$ of training to obtain 12 data points for each animal: one for each block of the five training sessions. The top four panels of Figure 2 show these data for four different response measures: change in response likelihood (percentage of CRs); CR magnitude; UR magnitude; and latency to onset (as measured by the latency to achieve the $0.3 \mathrm{~mm}$ CR criterion). These data reveal the magnitude and time course of the within-session changes. Separate two-way mixed ANOVAs (groups by blocks) for each of the four response measures revealed the following (Table 2, $F$ and $p$ values). For the response likelihood measure, there were significant main effects for groups, blocks, and group-by-block interaction, indicating significant within-session changes and differences in these changes between groups. The conditioned response magnitude and latency to onset analyses each displayed significant effects of blocks and group-by-block interaction, again indicating significant within-session changes that differed between groups. In contrast, there were no significant main or interaction effects for the UR amplitude data. This observation suggests that the changes in conditioned responses are not attributable to motor fatigue or within-session declines in the ability to measure the responses properly.
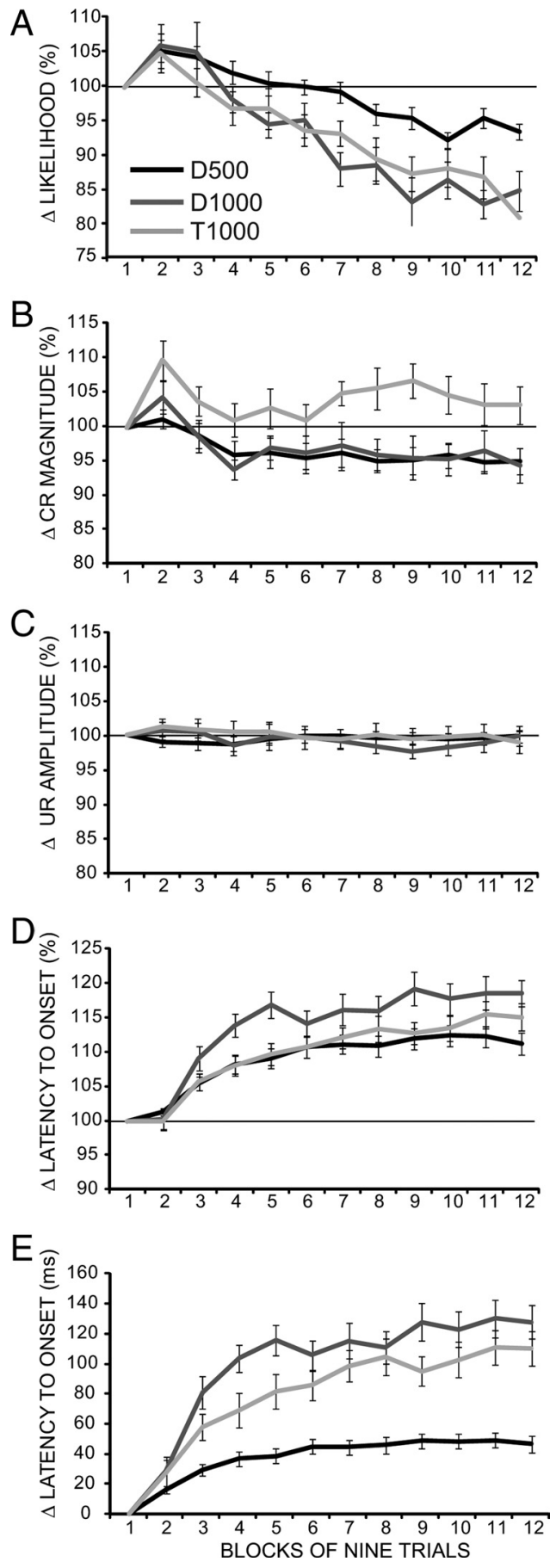

Figure 2. Analysis of within-session changes averaged over sessions 9 and 10 for five response measures for the same three groups shown in Figure 1. The abscissa shows the 12 nine-trial blocks that comprised each session. Each response measure involved an average of the nine trials for each block normalized to the average of the first block of that session, with data from sessions 9 and 10 then averaged to produce a single data point per animal per block. For the top four panels, the data were normalized as the percentage of the first block, whereas in the bottom panel the latency to onset was normalized as a difference score from the first block. $A$, There is a significant decrease within training sessions in response likelihood that is greater for the groups with an ISI of $1000 \mathrm{~ms}$. $\boldsymbol{B}$, There were small decreases in the magnitude of responses for the two delay conditioning groups. $C$ There were no significant changes in the amplitude of the URs. $\boldsymbol{D}$, There were robust increases in the latencies to onset of the conditioned responses for all three groups. $\boldsymbol{E}$, When the latency to onset data are normalized as a difference score the within-session increases are larger for the groups with an ISI of $1000 \mathrm{~ms}$. 
Table 2. ANOVA results for Figure 2 data

\begin{tabular}{lll}
\hline Response measure & Group main effect & Block main effect \\
\hline Degrees of freedom & 2,81 & 10,861 \\
Likelihood & $F=5.345, p=0.0066$ & $F=37.944, p<0.0001$ \\
Magnitude & $F=1.594$, NS & $F=18.232, p<0.0001$ \\
UR amplitude & $F=0.1313$, NS & $F=0.7702$, NS \\
Latency (ratio) & $F=2.606$, NS & $F=81.196, p<0.0001$ \\
Latency (difference) & $F=16.995, p<0.0001$ & $F=64.518, p<0.0001$ \\
\hline
\end{tabular}
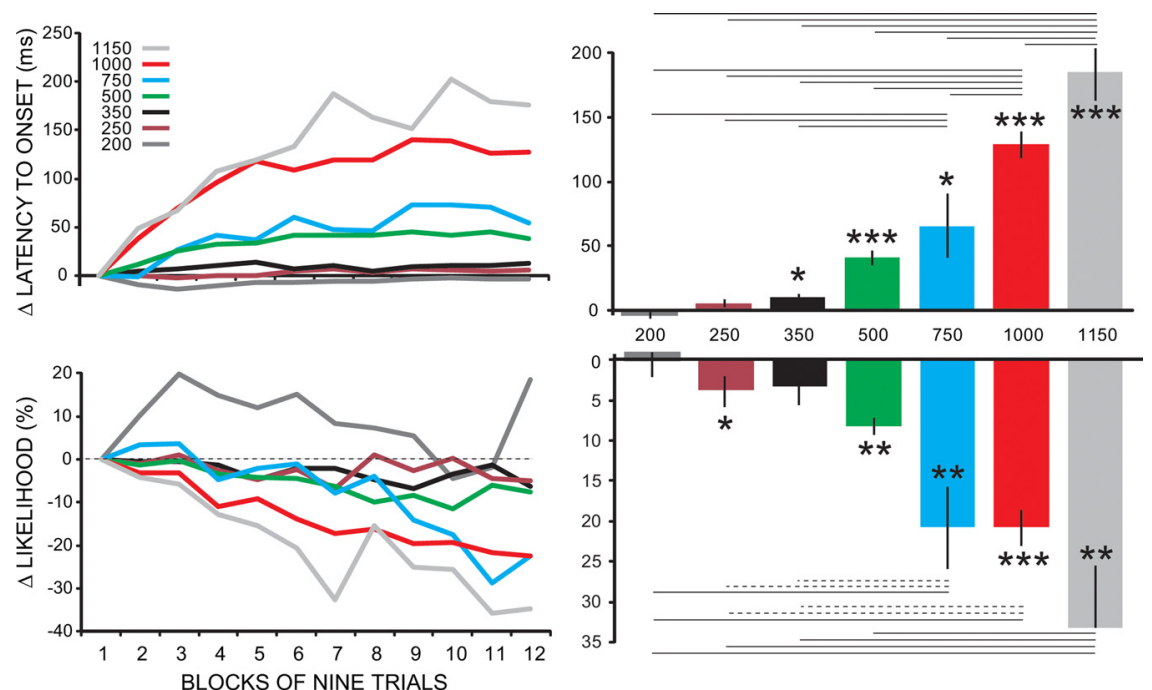

Figure 3. The effect of ISI on within-session changes of conditioned responses. Separate groups of animals were trained using ISIs of $200 \mathrm{~ms}(n=4), 250 \mathrm{~ms}(n=8), 350 \mathrm{~ms}(n=8), 500 \mathrm{~ms}(n=34), 750 \mathrm{~ms}(n=8), 1000 \mathrm{~ms}(n=21)$, and $1150 \mathrm{~ms}(n=$ 7). For the panels at left, the data were normalized as in Figure 2 (as a difference score relative to block 1 ) and averaged over the last two sessions of training for each animal (the total number of sessions varied from animal to animal, but performance was at asymptote for both). The graphs at right plot the same data as a single difference score (average of the last three blocks relative to first block) for all seven groups. There were significant increases in latency to onset and decreases in conditioned response likelihood that generally increased with the ISI. In the right panels, the asterisks denote significant differences from no change $\left({ }^{*} p<0.05,{ }^{* *} p<0.01,{ }^{* * *} p<0.001\right)$. The horizontal lines denote significant differences between groups (Tukey test), where dotted lines denote $p<0.05$ and solid lines denote $p<0.01$ ).

Because the mean onset latencies for the three groups were quite different, we performed an additional test on these data. Rather than normalizing as a ratio, an averaged difference score was obtained for the same $5 \mathrm{~d}$ (block score - block 1 for the same session). These results are shown in Figure 2E. A two-way mixed ANOVA revealed robust group, blocks, and group-by-block interaction effects (Table 2), indicating significant within-session changes in the timing of conditioned responses that differed across groups. Together, these data reveal that there are significant within-session changes in conditioned responses that are apparent at asymptotic performance and that reverse between sessions. Moreover, the significant group and group-by-block interaction effects indicate that the magnitude of these withinsession changes may depend upon the ISI. The following experiments tested this possibility more directly.

\section{Within-session changes increase with the ISI}

To evaluate the effect of ISI more thoroughly, we (1) added five additional delay conditioning groups (ISI $=200,250,500,750$, and $1050 \mathrm{~ms}$ ) to the D500 and D1000, (2) focused analysis only on delay conditioning, and (3) obtained a single difference score for each subject (for response likelihood $=$ mean over the last three blocks/mean of first block, averaged over last two sessions of training) (for latency to onset $=$ mean over the last three blocks - mean of first block, averaged over last two sessions of training) to facilitate individual post hoc comparisons across different groups.

As illustrated in Figure 3, this analysis revealed a clear ISI effect on the withinsession changes seen in both response measures. The changes in latency to onset and response likelihood increased as the ISI increased. A between-subjects ANOVA of the difference scores (end of session - beginning of session) revealed a robust statistical difference across the different ISI groups (latency to onset: $F_{(6,83)}=$ 29.35, $p<0.0001$; response likelihood: $\left.F_{(6,83)}=10.99, p<0.0001\right)$. Individual $t$ tests for each group revealed significant within-session changes in latency to onset for ISIs of $\geq 350$, and significant changes for response likelihood for ISIs of 250, 500, 750, 1000, and $1150 \mathrm{~ms}$. Pairwise comparisons of groups using the Tukey test revealed the patterns shown in the right half of Figure 3, where solid and dotted lines indicate a pairwise significance at $p<0.01$ and $p<0.05$, respectively. These data show a clear and monotonic increase in the within-session changes in likelihood and latency to onset as the ISI increased.

Within-subject differences depend on the type of training To appreciate the potential mechanistic implications of these within-session changes, it is necessary to address the possible contributions of sensory adaptation, response fatigue, or other generalized changes in the motor pathways downstream of the cerebellum. To address these important issues, we analyzed data from 49 rabbits trained for 10 daily sessions with a dual delaytrace conditioning procedure (Kalmbach et al., 2009). In this procedure, two different tones ( 1 and $9.5 \mathrm{kHz})$ are used as CSs. One CS is used for delay conditioning (ISI $=500$ ) and the other for trace conditioning trials identical to those used in the results of Figure 1 (500 ms CS, 1000 ms ISI). Each 108 trial training session was composed of a pseudorandom sequence of delay and trace trials such that each session involved 54 trials of each type, and each block involved at least four trials of each type. As shown in Figure 4, these procedures give rise to acquisition of robust responses to each CS with latencies to onset appropriate for each ISI (responses timed to peak near US onset in each case). These data replicate, in a within-subject comparison, the differences between D500 and T1000 (Fig. 1) and the effects of ISI on within-session changes (Fig. 3). Calculating difference scores in the manner used for Figure 3, revealed (1) statistically significant within-session changes for both delay and trace responses $(p<0.001$ for all measures except the magni- 
tude measure for D500, which was $p>$ 0.05 ), and (2) statistically significant differences in the changes for D500 versus T1000 for all three response measures (paired $t$ test, $p<0.0001$ in each case).

These data show that the magnitude of the changes in response likelihood and latency to onset can differ for the same subject and the same session depending on the type of training or ISI. This pattern of results cannot be explained by motor fatigue. Assuming that the eyelid responses to the two CSs are mediated by the same deep cerebellar nucleus neurons (Kalmbach et al., 2009) and downstream motor pathways, as suggested by the anatomy of the cerebellum, these data also suggest that the neural changes that mediate the within-session changes in conditioned responses do not occur downstream of the cerebellum. The next experiment addressed the potential for sensory adaptation or changes upstream of the cerebellum.

\section{Within-session changes using} stimulation of mossy fibers as the CS As mentioned in the Introduction, the ability to electrically stimulate mossy fibers to substitute for a CS is a key component of the evidence that eyelid conditioning is mediated by the cerebellum and that the behavioral properties of eyelid conditioning reflect a first approximation of the cerebellar rules for input-to-output transformations. Specifically, the ability of mossy fiber stimulation to serve as a CS excludes the necessity for plasticity upstream from the cerebellum. To conduct a similar test for the locus of the plasticity mediating the within-session changes in conditioned responses, we analyzed 35 animals that were trained using mossy fiber stimulation as the CS, 27 using an ISI of $500 \mathrm{~ms}$ (MF500), and 8 of $1000 \mathrm{~ms}$ (MF1000). Focusing on the last $2 \mathrm{~d}$ of training for each animal, which was within the asymptotic performance phase for each, we generated the same normalized and difference scores used for the analyses of Figures 3 and 4, respectively. As shown in Figure 5, the data reveal the following: (1) within-session changes in response likelihood, magnitude (data not shown) and latency to onset were apparent; (2) the changes were larger for MF1000 than for MF500; and (3) the effects in each case were no different from the corresponding changes for a tone CS at the same ISI. A two-way, mixed ANOVA on the normalized block data for the four groups (D500, D1000, MF500, and MF1000) revealed significant main effects of group (latency to onset: $F_{(3,83)}=3.306, p=0.02415$; response likelihood: $F_{(3,83)}=3.341, p=0.0212$ ), and blocks (latency to onset: $F_{(10,833)}=44.22, p<0.0001$; response likelihood: $\left.F_{(10,833)}=18.22, p<0.0001\right)$ as well as significant group-byblock interactions. These analyses indicate that there were significant within-session changes in both measures that differed among the four groups.
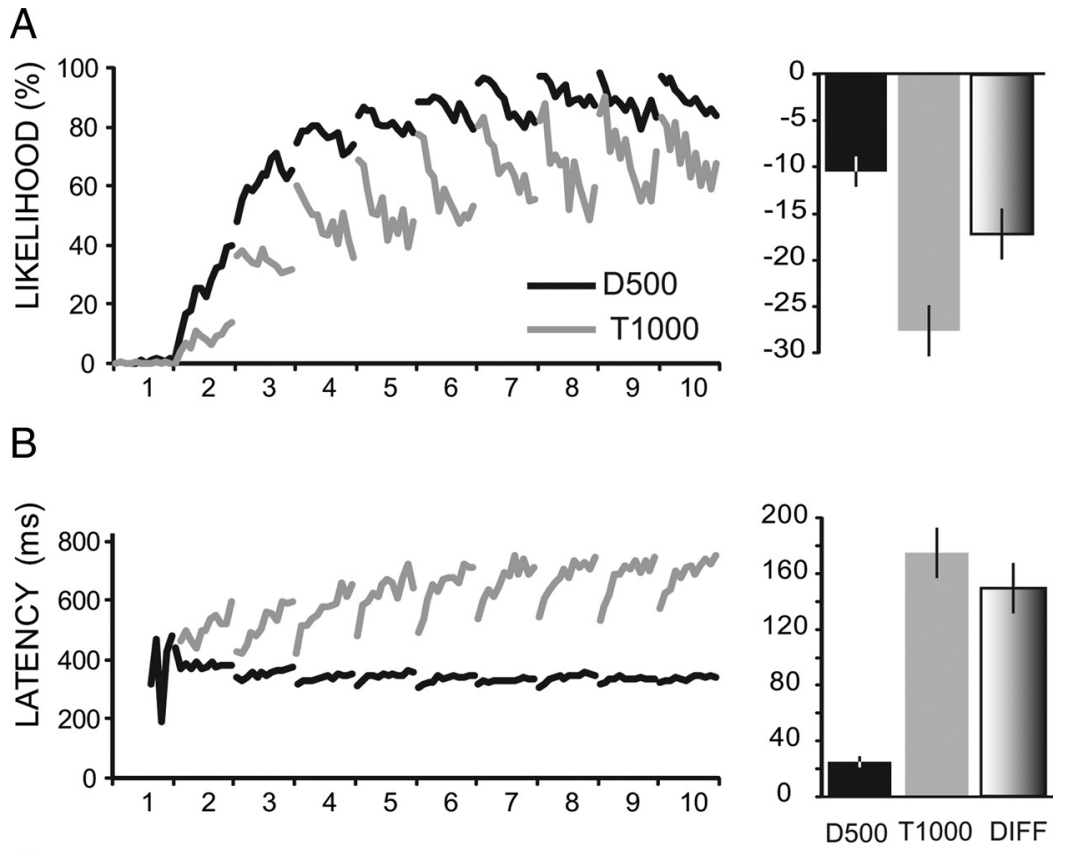

4. A within-subject comparison $(n=49)$ of the within-session changes for animals trained using two CSs, one trained with delay conditioning (D500) and the other trained with trace conditioning (T1000). The delay and trace trials were intermixed uring the sessions and then subjected to separate analyses. $\boldsymbol{A}-\boldsymbol{C}$, For response likelihood $(\boldsymbol{A})$, latency to onset $(\boldsymbol{B})$, and response 列 作 9 and 10. For all three response measures and for both trial types, the within-session changes were significant ( $p<0.05$ for comparison to 0 ). All three responses measures showed significant differences between the two trial types, as indicated by difference scores that were reliably different from 0 ( $p<0.01$ in each case).

To facilitate evaluation of individual groups and pairwise comparisons among the groups, we again generated a single difference score for each subject as described above. The results for latency to onset and response likelihood are shown in the right portion of Figure 5. The first question we addressed was whether there was a significant within-session change for each of the four groups. Since these are difference scores, we used individual $t$ tests to determine whether each response measure was significantly different from zero (zero indicating no change within the session). Both response measures for all four groups were reliably different from zero $(p<0.0001$ for each). The next question concerned the potential differences among the four groups. We began with a one-way ANOVA, which showed reliable differences among the four groups (latency to onset: $F_{(3,88)}=19.96, p<0.0001$; likelihood: $F_{(3,88)}=$ $7.75, p=0.00012)$. Pairwise comparisons using the Tukey test revealed a straightforward pattern: comparisons of different ISIs were significantly different (MF1000 or D1000 vs MF500 vs D500, $p<0.05$ ) and comparisons between the same ISIs were not reliably different (MF500 vs D500 or MF1000 vs D1000, $p>0.05)$. 

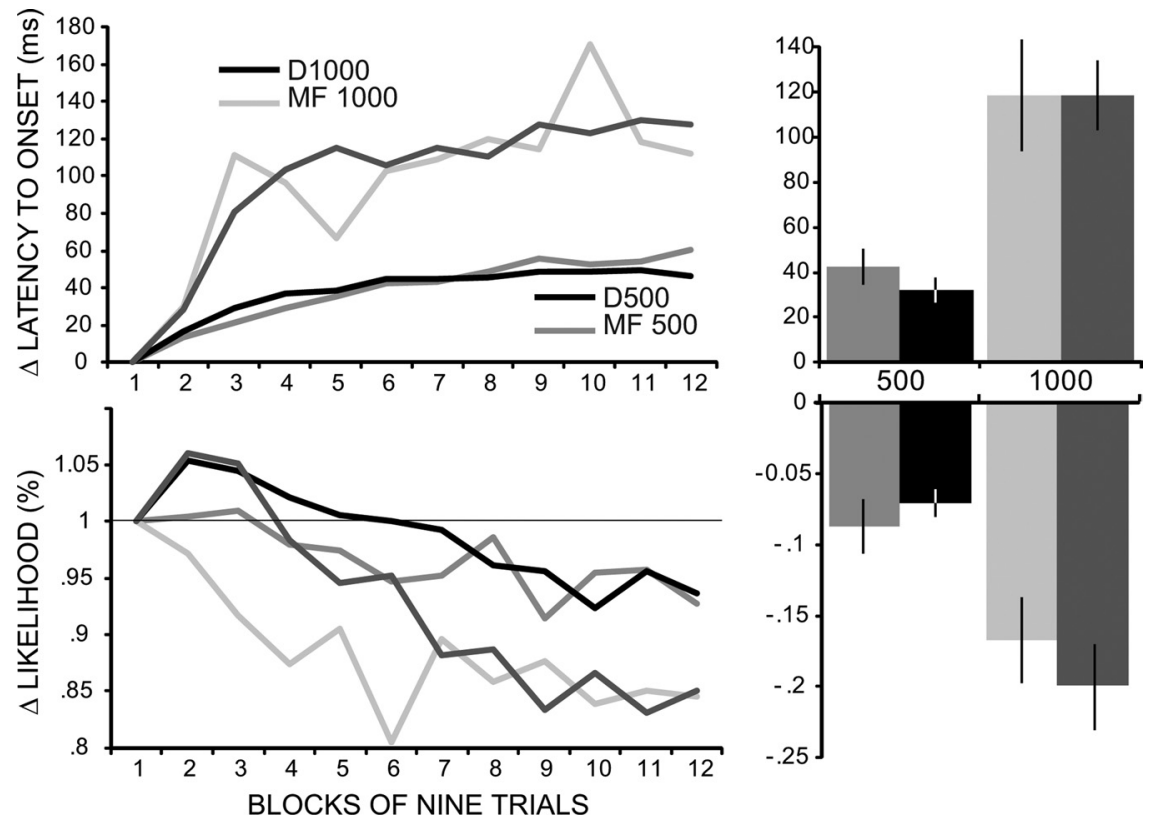

Figure 5. Using electrical stimulation of mossy fibers as a substitute for the tone CS also produced within-session changes in conditioned responses that were greater for an ISI of 1000 (MF1000, $n=8$ ) than for an ISI of 500 ms (MF500, $n=27$ ). For comparison purposes, data from animals using a tone CS (D500 and D1000, taken from Fig. 2) are also shown. These data, which are averages from sessions 9 and 10, are again normalized either as a percentage of block 1 (likelihood data) or as a difference score relative to block 1 (latency to onset data). Bar graphs at right show the same data expressed as a single normalized score (last three blocks relative to first block) averaged over sessions 9 and 10. Results from mossy fiber stimulation parallel those for a tone $C S$ : there are larger differences in response likelihood and latency to onset for the longer ISI. In the bar graphs, all four groups show reliable differences from zero change ( $p<0.01$ ). All comparisons between the same ISI were not significant, and all comparisons between different ISIs were reliably different $(p<0.05)$.

These results demonstrate that the within-session changes in conditioned responses are similar for a tone CS and a mossy fiber stimulation CS. For both types of CSs, the changes are greater for ISI $=1000$ than for ISI $=500$. These results exclude mechanisms upstream of the cerebellum as being necessary for the within-session changes. Combined with the previous experiment, these results suggest that changes neither upstream nor downstream from the cerebellum are necessary for the within-session changes in responding. From this, we conclude that changes within the cerebellum are sufficient to account for these behavioral phenomena. Having characterized the behavioral phenomena, explored the factors affecting their magnitude, and identified the cerebellum as the likely locus of the underlying mechanism, in a final study we used a large-scale computer simulation of the cerebellum to evaluate the candidate mechanisms. We next describe these efforts in some detail.

\section{Computer simulations of the cerebellum}

The extinction-like nature of the within-session changes characterized above offer hints as to the possible underlying processes within the cerebellum. Decrements in conditioned response magnitude and likelihood are certainly consistent with an extinction-like process. The within-session increases in onset latencies are perhaps even more telling. Previous work demonstrated that the timing of conditioned responses is enhancedthat is, the onset latencies are increased-by extinction-like processes within the cerebellum (Medina et al., 2000). Because evidence suggests that extinction is mediated by the increased strength of the CS-activated granule cell to Purkinje cells synapses
(Medina et al., 2000, 2001, 2002; Mauk and Ohyama, 2004), we used a computer simulation of the cerebellum to test the feasibility that decrementing changes in these synapses could explain the withinsession changes in conditioned responses. Testing this possibility does not require a specific commitment about how such changes may occur at granule to Purkinje cell synapses. Both presynaptically and postsynaptically expressed LTPs have been identified at the granule to Purkinje synapses (Hirano, 1990; Salin et al., 1996; Lev-Ram et al., 2002, 2003; Jörntell and Hansel, 2006). There could be a decrementing component to either process. Alternatively, the presynaptic form of LTP, where no presynaptic LTD counterpart has been identified, may reverse with a time course $>1$ and $<23 \mathrm{~h}$. Either way, we tested computers simulations of the cerebellum in which there was a decrementing form of LTP in addition to the known forms of long-lasting LTP and LTD.

Identical simulations, each implementing a form of activity-dependent decrementing plasticity at the granule to Purkinje synapses, were trained for five sessions using the same seven ISIs used for the rabbits studies $(200,250,350,500$, $750,1000,1150 \mathrm{~ms})$. In between each session, 5,000,000 time steps were run with background mossy fiber activity to emulate the time between training sessions for the rabbits. During this time, the value for the $\mathrm{dP}$ for each synapse gradually decayed to zero. Each simulation displayed learning of well timed "responses" and the responses from the last two sessions were analyzed in the same manner as shown for the data in Figure 3. As shown in Figure $6 A$, these simulations showed within-session declines in response amplitude and in latency to onset. As with the rabbits, these effects increased with the ISI because of the greater opportunities for $\mathrm{dP}$ induction with the longer CS. The simulation results did not properly mimic declines in response likelihood within sessions. We have observed in a variety of circumstances that the simulation better matches learned changes in amplitude rather than changes in response likelihood (other than that, likelihood goes up during acquisition and down during extinction). This failure, therefore, appears to be a general shortcoming of the simulation, rather than a specific shortcoming of short-term LTP, to explain within-session decreases in conditioned response likelihood.

These same simulations were then repeated using four different maximum values obtainable for the $\mathrm{dP}$ (Fig. $6 \mathrm{~B}$ ). With a maximum value of zero (i.e., no $\mathrm{dP}$ ), latencies to onset and response amplitudes were relatively stable over the 12 blocks of a training session. With increasing maximum values for $\mathrm{dP}$, the magnitude of the within-session effects also increased. These results indicate that implementing a decrementing form of potentiation at the granule to Purkinje synapses may be sufficient to explain the within-session changes in conditioned responses seen in the rabbits and that the only adjustment required for a quantitative match is the proper value for the maximum level this potentiation can obtain (Fig. $6 B$ ). 
In performing these tests, one unknown was whether the induction of this decrementing plasticity is controlled or prevented by climbing fiber inputs. The biological plausibility of the latter possibility relates to the clear evidence for endocannabinoid-mediated retrograde communication between Purkinje cells and the presynaptic elements of granule to Purkinje synapses (Brown et al., 2003, 2004; Safo and Regehr, 2005, 2008). There is also clear evidence that climbing fiber inputs can influence this communication (although there is no evidence that endocannabinoids can influence the induction of presynaptic LTP) (Safo and Regehr, 2005, 2008). Our solution to this unknown was to run identical simulations that differed only by the property that the induction of the $\mathrm{dP}$ was prevented for activity that occurred in the $100 \mathrm{~ms}$ following the arrival of a climbing fiber input (i.e., the time interval over which the same activity would engage long-term LTD at the same synapse). As shown in Figure 6C, this change produced results very similar to the previous simulations. The withinsession effects on latency to onset were almost identical to the previous runs (data not shown), and there was only a slight reduction in the within-session decreases in response amplitude that was most notable for the longer ISIs. Although this pattern matches the rabbit results slightly better-more robust changes in latency to onset than likelihood or magnitude-the subtleness of this effect demonstrates that the qualitative prediction is independent of any assumptions about dependence or independence of this process on climbing fiber inputs.

In separate simulations, we tested decrementing forms of plasticity at synapses that could, in principle, lead to withinsession decreases in conditioned response amplitude. In one set of simulations a decrementing form of LTD was included at the mossy fiber synapses onto the deep nucleus cells, and another set included decrementing LTD at the granule cell synapses onto the stellate and basket cells. Although it was possible in both cases to increase the maximum value of this plasticity to the point of producing small within-session decreases in response amplitude, neither produced measurable changes in the latency to onset of the responses (data not shown). These results certainly do not rule out contributions from these or other synapses, as this would overstate the scope of what can be accomplished using simulations. We cannot, for example, rule out that different implementations of these forms of plasticity could be more effective, nor can we exclude the possibility that the failure of these forms of plasticity simply arise from inaccuracies in the isomorphism between the simulations and the cerebellum. We simply point to these negative results and the ease with which the behavioral findings can be matched by implementing LTP at the granule to Purkinje synapses as our basis for favoring the latter as a candidate explanation.
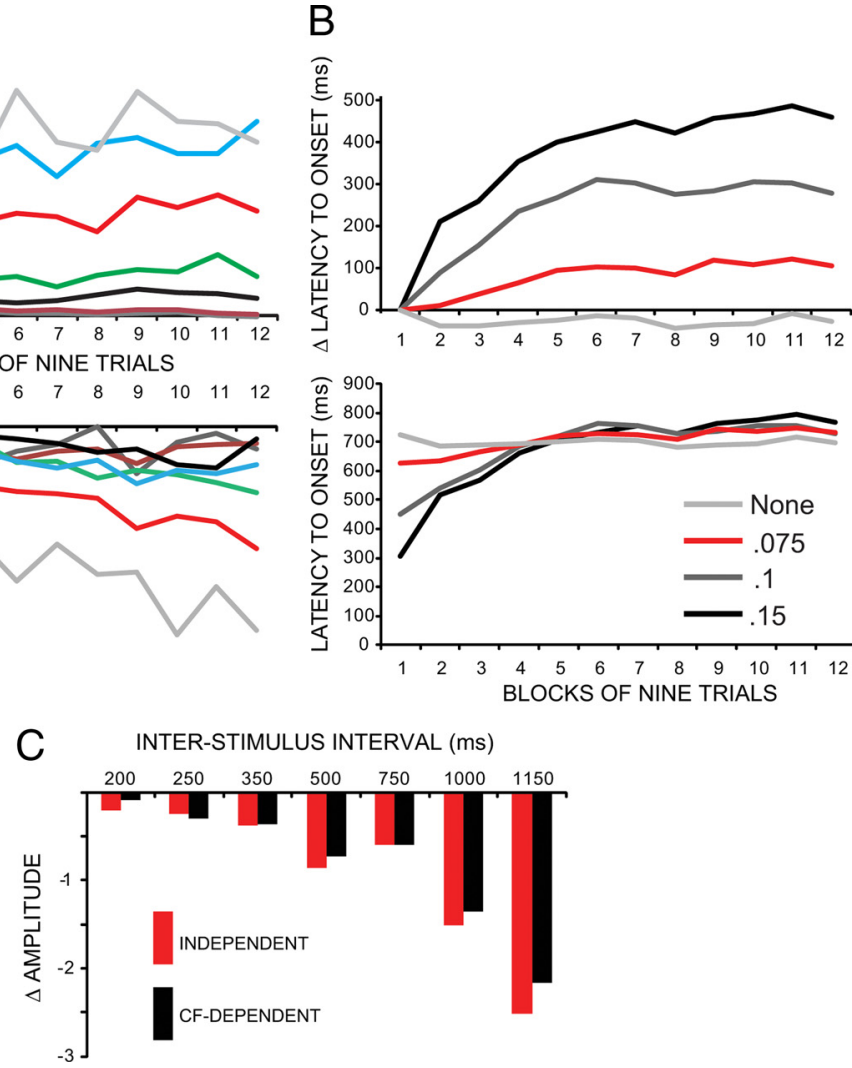

puter simulation of the cerebellum subjected to training using the same seven ISIs used whe rabbit experiments. When a decrementing form of potentiation (dP) is implemented at the granule to Purkinje decreases in conditioned response magnitude. $\boldsymbol{B}$, The magnitude of the within-session changes scales with the maximum obtainable value of $d P$. Without $d P$, both response measures are relatively stable within sessions. The red lines show results The changes in latency to onset were essentially identical (data not shown), and, aside from slightly smaller effects at itude were quite similar.

\section{Discussion}

We have documented within-session changes in the properties of conditioned eyelid responses that reverse before the next session. These changes increase with the ISI and are consistent with the buildup of an extinction-like process within the cerebellum. Specifically, there is a decrease in the likelihood and magnitude of the conditioned responses and an increase in their latencies to onset. Two sets of observations combine to suggest that these behavioral phenomena are mediated by changes occurring within the cerebellum. Replacing the tone CS with electrical stimulation of mossy fiber inputs to the cerebellum produces similar withinsession changes. This excludes sites upstream of the cerebellum, including nonspecific effects such as sensory adaptation. Conversely, the finding that the magnitude of the within-session changes can differ significantly within the same animal and session depending on the type of training suggests that changes downstream of the cerebellum can also be excluded. Assuming that delay and trace eyelid responses engage the same anterior interpositus neurons (Kalmbach et al., 2009), mechanisms acting downstream of the cerebellum would require similar behavioral changes for all manipulations within any given session. These same data, along with the lack of within-session changes in UR 
amplitude (Fig. 2), also permit exclusion of motor fatigue and other nonspecific changes as plausible explanations. From this set of observations, we infer that a decrementing form of activitydependent plasticity operates within the cerebellum and is engaged by delay and trace eyelid conditioning. In this instance, "decrementing" means a plasticity process that last for at least tens of minutes but for $<23 \mathrm{~h}$.

Although these results do not precisely indicate the location or nature of the underlying neural changes, they do impose constraints. The processes that change must be activity-dependent and engaged in a CS-specific manner by mossy fiber inputs to the cerebellum. These conclusions follow from the observations that the behavioral effect sizes are specific to the training for the relevant CS and from the ability of a mossy fiber stimulation CS to produce similar effects. Any viable hypothesis regarding the location and nature of the underlying neural processes must also explain how they are engaged to a greater extent by longer conditioned stimuli than by shorter ones. This is suggested by the monotonic relationship between interstimulus interval and the magnitude of the within-session behavioral effects. We can also tentatively infer that the underlying neural changes may be related to, or interact with, processes of extinction within the cerebellum.

Based on these constraints, we hypothesize the involvement of an LTP-like process ( $\mathrm{dP})$ operating at the granule to Purkinje synapses that lasts for at least tens of minutes but $<23 \mathrm{~h}$. To test the plausibility of this mechanism in explaining the behavioral effects, we implemented a decrementing form of potentiation at the granule to Purkinje cells synapses of a large-scale computer simulation of the cerebellum that reproduces the basic behavioral properties of eyelid conditioning. Both the ability of such a simulation to reproduce the within-session changes in responding, including their dependence on the ISI, and the straightforward scaling of the behavioral effects with the maximum allowed value of $\mathrm{dP}$ support the plausibility of $\mathrm{dP}$ as a mechanistic basis for the behavioral observations.

Although the simulation results are consistent with the involvement of LTP at the granule to Purkinje synapses, they certainly do not constitute proof that it is the underlying basis for within-session changes in eyelid responses. Several forms and sites of plasticity have been identified in the cerebellum, and there are other synapses that cannot be excluded (Hansel et al., 2001). Forms of short-term plasticity have been characterized at cerebellar synapses including a short-term facilitation and depression at the mossy fiber to granule synapses (Nieus et al., 2006), an associative short-term depression at granule to Purkinje synapses (Beierlein et al., 2007), and a lone report of a short-term potentiation (lasting tens of minutes) at the granule to Purkinje synapses (Goto et al., 2006). Thus, there are many possibilities, both discovered and perhaps undiscovered, that might contribute to the within-session changes. With the exception of the lone report of a facilitation lasting tens of minutes at the granule to Purkinje synapses, all other forms of short-term plasticity reported at cerebellar synapses appear to be too short lasting to explain our observations.

Based on available evidence, it is not possible to exclude any of the long-term forms of plasticity that have been identified in the cerebellum because in each case "long-term" is defined as lasting an hour or two in vitro. Our results would be entirely consistent with one of these forms of plasticity should its actual duration in vivo turn out to be $<23 \mathrm{~h}$. In vitro experiments rarely monitor the expression of the presynaptically expressed form of LTP at the granule to Purkinje synapses for more than $1 \mathrm{~h}$. As such, there is no evidence, to our knowledge, that excludes the possibility that this form of plasticity fades with a time course of 2-20 h. One factor that makes this possibility intriguing is that no presynaptic LTD has been identified at the granule to Purkinje synapse. Assuming that all forms of plasticity must have some mechanism of reversal, irreversible saturation being the eventually consequence otherwise, it remains possible that presynaptic LTP at these synapses is reversed by time rather than by a complementary LTD process.

However intriguing these possibilities may seem, additional experiments will be required to exclude the many viable alternatives. Nevertheless, our results strongly suggest that such a process exists within the cerebellum and that its contributions must be a part of a complete understanding of the mechanism for learning and information processing in the cerebellum.

This leads to the question of what specific contributions a decrementing plasticity process might make to cerebellar information processing. At this point, only speculations are possible as our data address only the presence and possible location of such a process. Preliminary simulation work suggests the possibility that it could contribute to resistance to forgetting. As time elapses since the last training trial, there are incremental opportunities for forgetting caused by plasticity at the synapses encoding the conditioned response. Decrementing LTP could represent a hedge against this by leading to more LTD, which later, after some degradation, would support a response whose amplitude is closer to the proper level than it would have been otherwise. When proper in balance, these processes would not be apparent behaviorally: the extra LTD during training would be counteracted by the decrementing LTP and later, forgetting would be counteracted by the extra LTD and the time-dependent decline of the LTP. This may explain the ISI-dependent increase in the behavioral effects, whereas in more natural behaviors such as vestibulo-ocular reflex adaptation the processes might be in better balance and thus more hidden. As with the question of the location and nature of the underlying decrementing processes, further experiments will be required to test this hypothesis.

\section{References}

Abbott LF, Nelson SB (2000) Synaptic plasticity: taming the beast. Nat Neurosci [3 Suppl]:1178-1183.

Aitkin LM, Boyd J (1978) Acoustic input to the lateral pontine nuclei. Hear Res 1:67-77.

Barbour B (1993) Synaptic currents evoked in Purkinje cells by stimulating individual granule cells. Neuron 11:759-769.

Beierlein M, Fioravante D, Regehr WG (2007) Differential expression of posttetanic potentiation and retrograde signaling mediate targetdependent short-term synaptic plasticity. Neuron 54:949-959.

Best AR, Regehr WG (2009) Inhibitory regulation of electrically coupled neurons in the inferior olive is mediated by asynchronous release of GABA. Neuron 62:555-565.

Boyd J, Aitkin L (1976) Responses of single units in the pontine nuclei of the cat to acoustic stimulation. Neurosci Lett 3:259-263.

Brickley SG, Cull-Candy SG, Farrant M (1996) Development of a tonic form of synaptic inhibition in rat cerebellar granule cells resulting from persistent activation of GABAA receptors. J Physiol 497:753-759.

Brown SP, Brenowitz SD, Regehr WG (2003) Brief presynaptic bursts evoke synapse-specific retrograde inhibition mediated by endogenous cannabinoids. Nat Neurosci 6:1048-1057.

Brown SP, Safo PK, Regehr WG (2004) Endocannabinoids inhibit transmission at granule cell to Purkinje cell synapses by modulating three types of presynaptic calcium channels. J Neurosci 24:5623-5631.

Buonomano DV (2000) Decoding temporal information: a model based on short-term synaptic plasticity. J Neurosci 20:1129-1141.

Buonomano DV, Maass W (2009) State-dependent computations: spatiotemporal processing in cortical networks. Nat Rev Neurosci 10:113-125. Buonomano DV, Mauk MD (1994) Neural network model of the cerebel- 
lum: temporal discrimination and the timing of motor responses. Neural Comput 6:38-55.

Cruikshank SJ, Weinberger NM (1996) Evidence for the Hebbian hypothesis in experience-dependent physiological plasticity of neocortex: a critical review. Brain Res Brain Res Rev 22:191-228.

D’Angelo E, De Filippi G, Rossi P, Taglietti V (1995) Synaptic excitation of individual rat cerebellar granule cells in situ: evidence for the role of NMDA receptors. J Physiol 484:397-413.

Dieudonné S (1995) Glycinergic synaptic currents in Golgi cells of the rat cerebellum. Proc Natl Acad Sci U S A 92:1441-1445.

Dieudonne S (1998) Submillisecond kinetics and low efficacy of parallel fibre-Golgi cell synaptic currents in the rat cerebellum. J Physiol 510:845-866.

Fisher SA, Fischer TM, Carew TJ (1997) Multiple overlapping processes underlying short-term synaptic enhancement. Trends Neurosci 20:170177.

Fortune ES, Rose GJ (2000) Short-term synaptic plasticity contributes to the temporal filtering of electrosensory information. J Neurosci 20:71227130 .

Fortune ES, Rose GJ (2002) Roles for short-term synaptic plasticity in behavior. J Physiol Paris 96:539-545.

Goto J, Inoue T, Kuruma A, Mikoshiba K (2006) Short-term potentiation at the parallel fiber-Purkinje cell synapse. Neurosci Res 55:28-33.

Gould TJ, Sears LL, Steinmetz JE (1993) Possible CS and US pathways for rabbit classical eyelid conditioning: electrophysiological evidence for projections from the pontine nuclei and inferior olive to cerebellar cortex and nuclei. Behav Neural Biol 60:172-185.

Hansel C, Linden DJ, D’Angelo E (2001) Beyond parallel fiber LTD: the diversity of synaptic and nonsynaptic plasticity in the cerebellum. Nat Neurosci 4:467-475.

Hesslow G, Svensson P, Ivarsson M (1999) Learned movements elicited by direct stimulation of cerebellar mossy fiber afferents. Neuron 24:179-185.

Hirano T (1990) Depression and potentiation of the synaptic transmission between a granule cell and a Purkinje cell in rat cerebellar culture. Neurosci Lett 119:141-144.

Ito M (2001) Cerebellar long-term depression: characterization, signal transduction, and functional roles. Physiol Rev 81:1143-1195.

Jörntell H, Hansel C (2006) Synaptic memories upside down: bidirectional plasticity at cerebellar parallel fiber-Purkinje cell synapses. Neuron $52: 227-238$

Kalmbach BE, Ohyama T, Kreider JC, Riusech F, Mauk MD (2009) Interactions between prefrontal cortex and cerebellum revealed by trace eyelid conditioning. Learn Mem 16:86-95.

Kaneda M, Farrant M, Cull-Candy SG (1995) Whole-cell and singlechannel currents activated by GABA and glycine in granule cells of the rat cerebellum. J Physiol 485:419-435.

Karmarkar UR, Buonomano DV (2007) Timing in the absence of clocks: encoding time in neural network states. Neuron 53:427-438.

Kehoe EJ, Gormezano I (1974) Effects of trials per session on conditioning of the rabbit's nictitating membrane response. Bull Psychon Soc 2:434-436.

Kehoe EJ, White NE (2002) Extinction revisited: similarities between extinction and reductions in US intensity in classical conditioning of the rabbit's nictitating membrane response. Learn Behav 30:93-111.

Kehoe EJ, Weidemann G, Darnall S (2004) Apparatus exposure produces profound declines in conditioned nictitating-membrane responses to discrete conditioned stimuli by the rabbit (Oryctolagus cuniculus) J Exp Psychol Anim Behav Process 30:259-270.

Konnerth A, Llano I, Armstrong CM (1990) Synaptic currents in cerebellar Purkinje cells. Proc Natl Acad Sci U S A 87:2662-2665.

Lang EJ, Sugihara I, Llinás R (1996) GABAergic modulation of complex spike activity by the cerebellar nucleoolivary pathway in rat. J Neurophysiol 76:255-275.

Lev-Ram V, Wong ST, Storm DR, Tsien RY (2002) A new form of cerebellar long-term potentiation is postsynaptic and depends on nitric oxide but not cAMP. Proc Natl Acad Sci U S A 99:8389-8393.

Lev-Ram V, Mehta SB, Kleinfeld D, Tsien RY (2003) Reversing cerebellar long-term depression. Proc Natl Acad Sci U S A 100:15989-15993.

Linden DJ (1994) Long-term synaptic depression in the mammalian brain. Neuron 12:457-472.
Llano I, Gerschenfeld HM (1993) Inhibitory synaptic currents in stellate cells of rat cerebellar slices. J Physiol 468:177-200.

Lynch MA (2004) Long-term potentiation and memory. Physiol Rev 84:87-136.

Maren S, Baudry M (1995) Properties and mechanisms of long-term synaptic plasticity in the mammalian brain: relationships to learning and memory. Neurobiol Learn Mem 63:1-18.

Martin SJ, Morris RG (2002) New life in an old idea: the synaptic plasticity and memory hypothesis revisited. Hippocampus 12:609-636.

Martin SJ, Grimwood PD, Morris RG (2000) Synaptic plasticity and memory: an evaluation of the hypothesis. Annu Rev Neurosci 23:649-711.

Mauk MD, Buonomano DV (2004) The neural basis of temporal processing. Annu Rev Neurosci 27:307-340.

Mauk MD, Donegan NH (1997) A model of Pavlovian eyelid conditioning based on the synaptic organization of the cerebellum. Learn Mem 4:130-158.

Mauk MD, Ohyama T (2004) Extinction as new learning versus unlearning: considerations from a computer simulation of the cerebellum. Learn Mem 11:566-571.

Mauk MD, Steinmetz JE, Thompson RF (1986) Classical conditioning using stimulation of the inferior olive as the unconditioned stimulus. Proc Natl Acad Sci U S A 83:5349-5353.

McCormick DA, Thompson RF (1984) Cerebellum: essential involvement in the classically conditioned eyelid response. Science 223:296-299.

Medina JF, Mauk MD (1999) Simulations of cerebellar motor learning: computational analysis of plasticity at the mossy fiber to deep nucleus synapse. J Neurosci 19:7140-7151.

Medina JF, Mauk MD (2000) Computer simulation of cerebellar information processing. Nat Neurosci [3 Suppl]:1205-1211.

Medina JF, Garcia KS, Nores WL, Taylor NM, Mauk MD (2000) Timing mechanisms in the cerebellum: testing predictions of a large-scale computer simulation. J Neurosci 20:5516-5525.

Medina JF, Garcia KS, Mauk MD (2001) A mechanism for savings in the cerebellum. J Neurosci 21:4081-4089.

Medina JF, Nores WL, Mauk MD (2002) Inhibition of climbing fibres is a signal for the extinction of conditioned eyelid responses. Nature 416:330-333.

Midtgaard J (1992) Membrane properties and synaptic responses of Golgi cells and stellate cells in the turtle cerebellum in vitro. J Physiol 457:329-354.

Miles FA, Lisberger SG (1981) Plasticity in the vestibulo-ocular reflex: a new hypothesis. Annu Rev Neurosci 4:273-299.

Mouginot D, Gähwiler BH (1995) Characterization of synaptic connections between cortex and deep nuclei of the rat cerebellum in vitro. Neuroscience 64:699-712.

Nieus T, Sola E, Mapelli J, Saftenku E, Rossi P, D’Angelo E (2006) LTP regulates burst initiation and frequency at mossy fiber-granule cell synapses of rat cerebellum: experimental observations and theoretical predictions. J Neurophysiol 95:686-699.

Ohyama T, Nores WL, Murphy M, Mauk MD (2003) What the cerebellum computes. Trends Neurosci 26:222-227.

Pugh JR, Raman IM (2006) Potentiation of mossy fiber EPSCs in the cerebellar nuclei by NMDA receptor activation followed by postinhibitory rebound current. Neuron 51:113-123.

Pugh JR, Raman IM (2008) Mechanisms of potentiation of mossy fiber EPSCs in the cerebellar nuclei by coincident synaptic excitation and inhibition. J Neurosci 28:10549-10560.

Pugh JR, Raman IM (2009) Nothing can be coincidence: synaptic inhibition and plasticity in the cerebellar nuclei. Trends Neurosci 32:170-177.

Puia G, Costa E, Vicini S (1994) Functional diversity of GABA-activated Clcurrents in Purkinje versus granule neurons in rat cerebellar slices. Neuron 12:117-126.

Safo P, Regehr WG (2008) Timing dependence of the induction of cerebellar LTD. Neuropharmacology 54:213-218.

Safo PK, Regehr WG (2005) Endocannabinoids control the induction of cerebellar LTD. Neuron 48:647-659.

Sakurai M (1987) Synaptic modification of parallel fibre-Purkinje cell transmission in in vitro guinea-pig cerebellar slices. J Physiol 394:463-480.

Salin PA, Malenka RC, Nicoll RA (1996) Cyclic AMP mediates a presynaptic form of LTP at cerebellar parallel fiber synapses. Neuron 16:797-803.

Shinoda Y, Sugiuchi Y, Futami T (1987) Excitatory inputs to cerebellar den- 
tate nucleus neurons from the cerebral cortex in the cat. Exp Brain Res 67:299-315.

Simpson JI, Wylie DR, de Zeeuw CI (1996) On climbing fibers signals and their consequences. Behav Brain Sci 19:384-398.

Stanton ME, Freeman JH Jr, Skelton RW (1992) Eyeblink conditioning in the developing rat. Behav Neurosci 106:657-665.

Steinmetz JE, Lavond DG, Thompson RF (1989) Classical conditioning in rabbits using pontine nucleus stimulation as a conditioned stimulus and inferior olive stimulation as an unconditioned stimulus. Synapse 3:225-233.

Stopfer M, Carew TJ (1996) Heterosynaptic facilitation of tail sensory neuron synaptic transmission during habituation in tail-induced tail and siphon withdrawal reflexes of Aplysia. J Neurosci 16:4933-4948.

Thompson RF, Steinmetz JE (2009) The role of the cerebellum in classical conditioning of discrete behavioral responses. Neuroscience 162:732-755.
Vincent P, Marty A (1996) Fluctuations of inhibitory postsynaptic currents in Purkinje cells from rat cerebellar slices. J Physiol 494:183-199.

Virginio C, Martina M, Cherubini E (1995) Spontaneous GABA-mediated synaptic currents in cerebellar granule cells in culture. Neuroreport 6:1285-1289.

Weidemann G, Kehoe EJ (2005) Stimulus specificity of concurrent recovery in the rabbit nictitating membrane response. Learn Behav 33:343-362.

Zhang W, Linden DJ (2006) Long-term depression at the mossy fiber-deep cerebellar nucleus synapse. J Neurosci 26:6935-6944.

Zucker RS (1989) Short-term synaptic plasticity. Annu Rev Neurosci 12:13-31.

Zucker RS, Regehr WG (2002) Short-term synaptic plasticity. Annu Rev Physiol 64:355-405. 